\title{
Respuesta a vismodegib en un meduloblastoma recurrente
}

\author{
Response to vismodegib in recurrent medulloblastoma
}

\author{
- Laura Bernal', Nicolás Useche2 , Sonia Bermúdez², Andrés Felipe Cardona ${ }^{1,3}$
}

' Clinical and Translational Oncology Group, Institute of Oncology, Fundación Santa Fe de Bogotá (Bogotá, Colombia).

2 Diagnostic Image Department, Neuro-Radiology Section, Fundación Santa Fe de Bogotá (Bogotá, Colombia).

${ }^{3}$ Foundation for Clinical and Applied Cancer Research (FICMAC); ONCOLGroup associate researcher.

A 32 year-old female patient was seen in 2006 suffering from headaches, vertical diplopia and a graduallydeveloping unsteady gait. CT scan revealed a $40 \times 40$ $\mathrm{mm}$ intra-axial cerebellar mass affecting the vermis. She underwent gross total resection with histology being compatible with a primitive neuro-ectodermal pattern. The final report was consistent with a desmoplastic nodular medulloblastoma (WHO grade IV), 20\% Ki67, with focal reactivity for synaptophysin, neurofilaments and CD99. Bone marrow biopsy and cerebrospinal fluid were negative. She received adjuvant craniospinal radiotherapy, followed by chemotherapy with cyclophosphamide, cisplatin, etoposide and vincristine which was well-tolerated. She was then closely followed-up for 5 years with no evidence of relapse until an MRI then identified a new lesion within the posterior fossa which was biopsied and confirmed recurrent disease. She was evaluated at MD Anderson Cancer Center (MDACC) and in conjunction with our oncology department we decided to treat her with induction chemotherapy involving cyclophosphamide, cisplatin and etoposide. After 6 cycles she underwent to intensified dose therapy (carboplatin, thiotepa and etoposide) achiving a complete response. Eleven-month postoperative MRI indicated tumour relapse which was confirmed by PET/ CT whilst complimentary 14-month imaging revealed leptomeningeal infiltration (figure 1). This was initially treated with gamma-knife radiosurgery, followed by one dose of bevacizumab. She tested positive for SHh with PATCHed-1 activation by immunohistochemistry; after this result we switch to $150 \mathrm{mg} /$ day vismodegib and the MRI revealed partial response after two months' treatment (figure 2). Her symptoms improved as well as her neurological exam.

Medulloblastomas (MB) are primary malignant embryonic tumours affecting the central nervous system (CNS). They are the most common CNS tumour in the paediatric population, but are very rare in the adult population ( 0,5 per million reported incidence), accounting for less than $1 \%$ of primary brain tumours in young adults ${ }^{1}$. Clinical features include truncal ataxia, gait disturbances and symptoms arising from increased intracranial pressure (headache, vomiting). Most originate within the posterior fossa, sometimes infiltrating across the ependymal lining into the brainstem or disseminating within central spinal fluid. Most information regarding its treatment is extrapolated from paediatric data or is based on retrospective reviews. Patients having recurrent disease after primary therapy have a particularly poor prognosis, mean survival rate (SR) being less than 6 months, 9\% having a 2-year SR2. Second line therapy is limited. Genome-wide sequencing has identified four distinct molecular subtypes: the wingless signalling (WNT) and sonic hedgehog pathway-activated groups ( $\mathrm{SHH}$ ), group 3 and group 4; $\mathrm{SHH}$ is the most common subtype, accounting for $60 \%$ to $80 \%$ of all adult MB. These medulloblastomas are often desmoplastic ${ }^{3}$.

The hedgehog signalling pathway is an embryonic signalling cascade which regulates stem cell differentiation; it is activated by transmembrane protein smoothened (SMO) signalling; the protein patched homologue 1 (PTCH1), sonic hedgehog receptor, suppresses hedgehog pathway activation by inhibiting SMO. SHH binding to PTCH1 results in inactivation of PTCH1, thereby limiting SMO inhibition and promoting pathway activation ${ }^{2,4,5}$. Mutations resulting in $\mathrm{SHH}$ over-activation are thus critical in the pathogenesis of various malignancies, including $M B$, providing an opportunity for targetted therapy. Cyclopamine (Veratrum alkaloid) was the first agent discovered to be able to disrupt this pathway by binding to $\mathrm{SMO}^{6,7}$, demonstrating in vitro and in vivo inhibition 
of tumour growth. This finding triggered active research into developing other SMO inhibitors.

The first results in humans demonstrating a response to the SMO inhibitor (GDC-0449) were published in 2009; a phase I clinical trial reported an acceptable safety profile and encouraging anti-tumour activity in advanced basal cell carcinoma (BCC) and $\mathrm{MB}^{8}$. Rapid tumour regression was seen in metastatic $\mathrm{MB}^{2}$. Such trials culminated in recent FDA approval of vismodegib for BCC in January 2012. Although trials are still ongoing, SMO inhibitors seem promising regarding MB. Phase II trial results evaluating vismodegib efficacy and safety in adult patients suffering recurrent or refractory MB medulloblastoma have been presented recently. Thirty-two patients having measureable disease were treated with vismodegib. No response was observed in non-SHH tumours. Three out of twenty patients having $\mathrm{SHH}$ tumours had a sustained response (maintained for at least 8 weeks). Mean therapy duration was 2,76 months; some patients remained on treatment for up to 13 months. The most common toxicity was grade 3 decrease in lymphocytes, myalgia, seizures, back pain and syncope, presented by 4 patients during a first course and 3 patients during a second one. One patient experienced a grade 4 thromboembolic event. These results demonstrated vismodegib activity and security concerning recurrent or refractory SHH subtype medulloblastoma9. At least 7 additional SMO antagonists are currently being developed in various stages of clinical trial ${ }^{10}$.

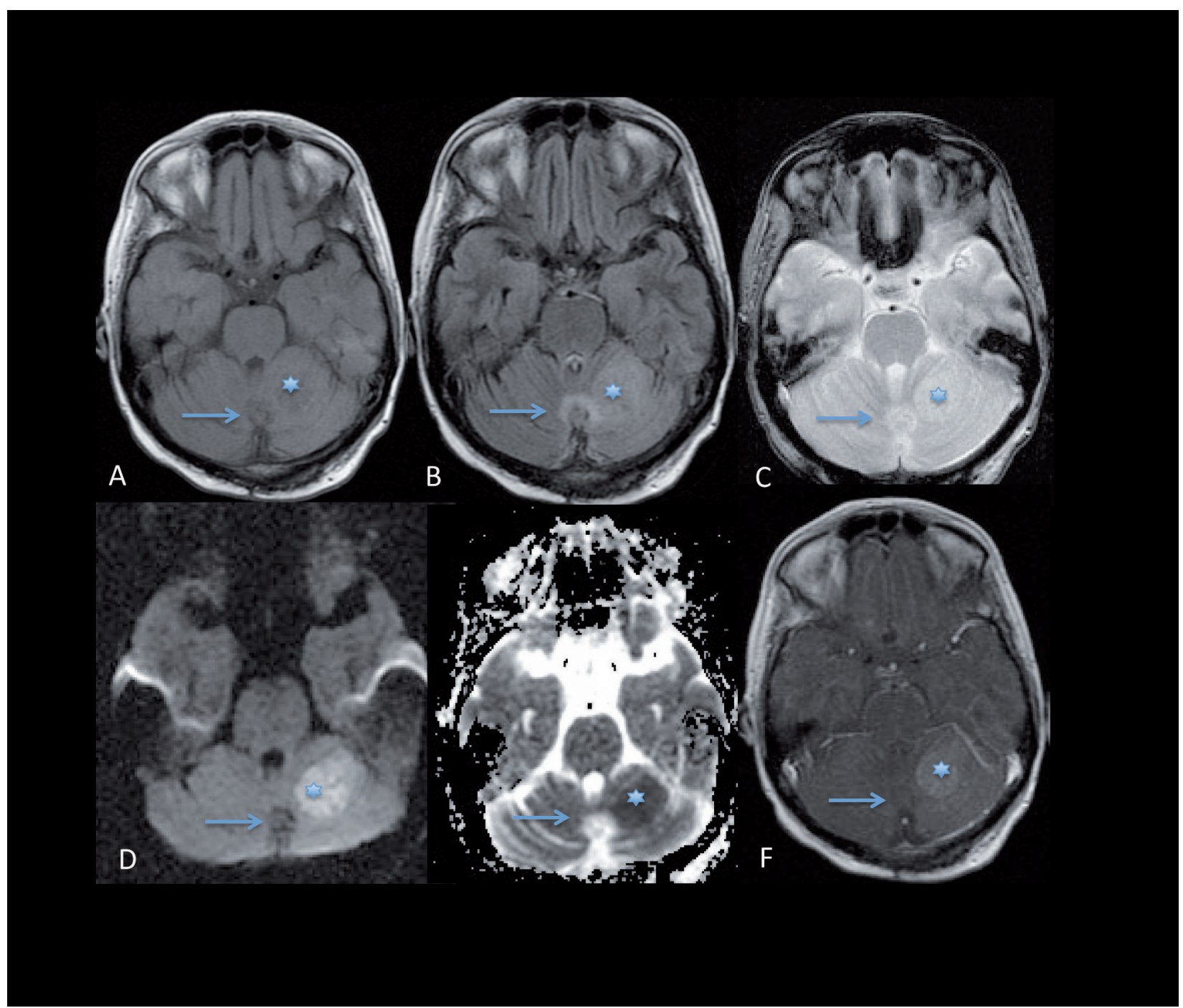

Figure 1. Simple and contrast magnetic resonance of the brain at the moment of relapse (2011)

A. TIW B. FLAIR C. GRET2 D. DWI E. ADC F.T1W Contrast

Axial cross-sections showing post-surgery encephalomalacy and gliosis in the vermis (arrow) and left cerebellum nodular lesion, revealed with contrast, without bleeding and with restriction of diffusion, showing tumour recurrence ${ }^{*}$. 


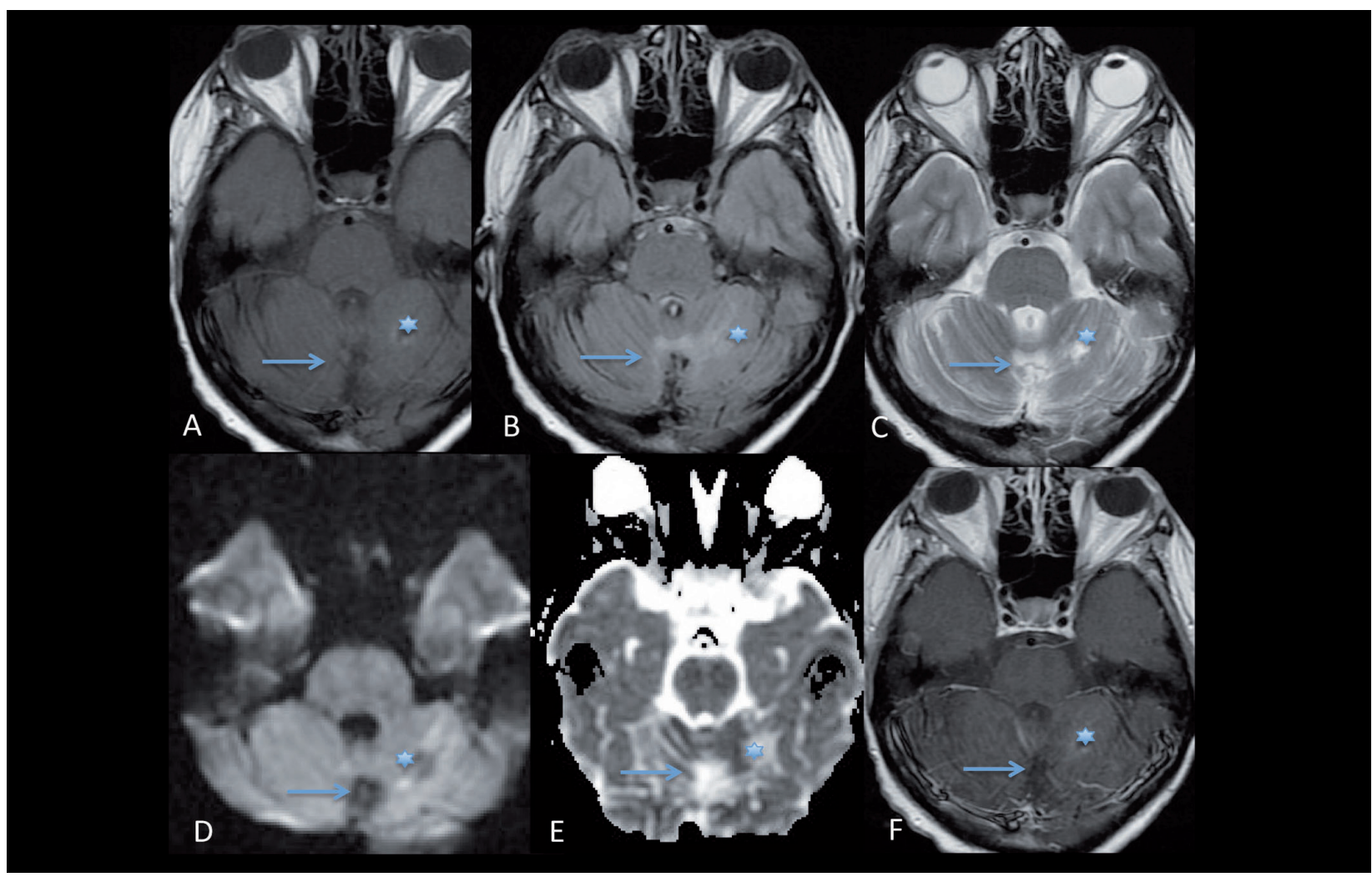

Figure 2. Simple and contrast magnetic resonance of the brain 6 months after high dose chemotherapy plus peripheral blood stem cell rescue (2012).

A.TIW B.FLAIR C. T2W D. DWI E.ADC F.TTW contrast

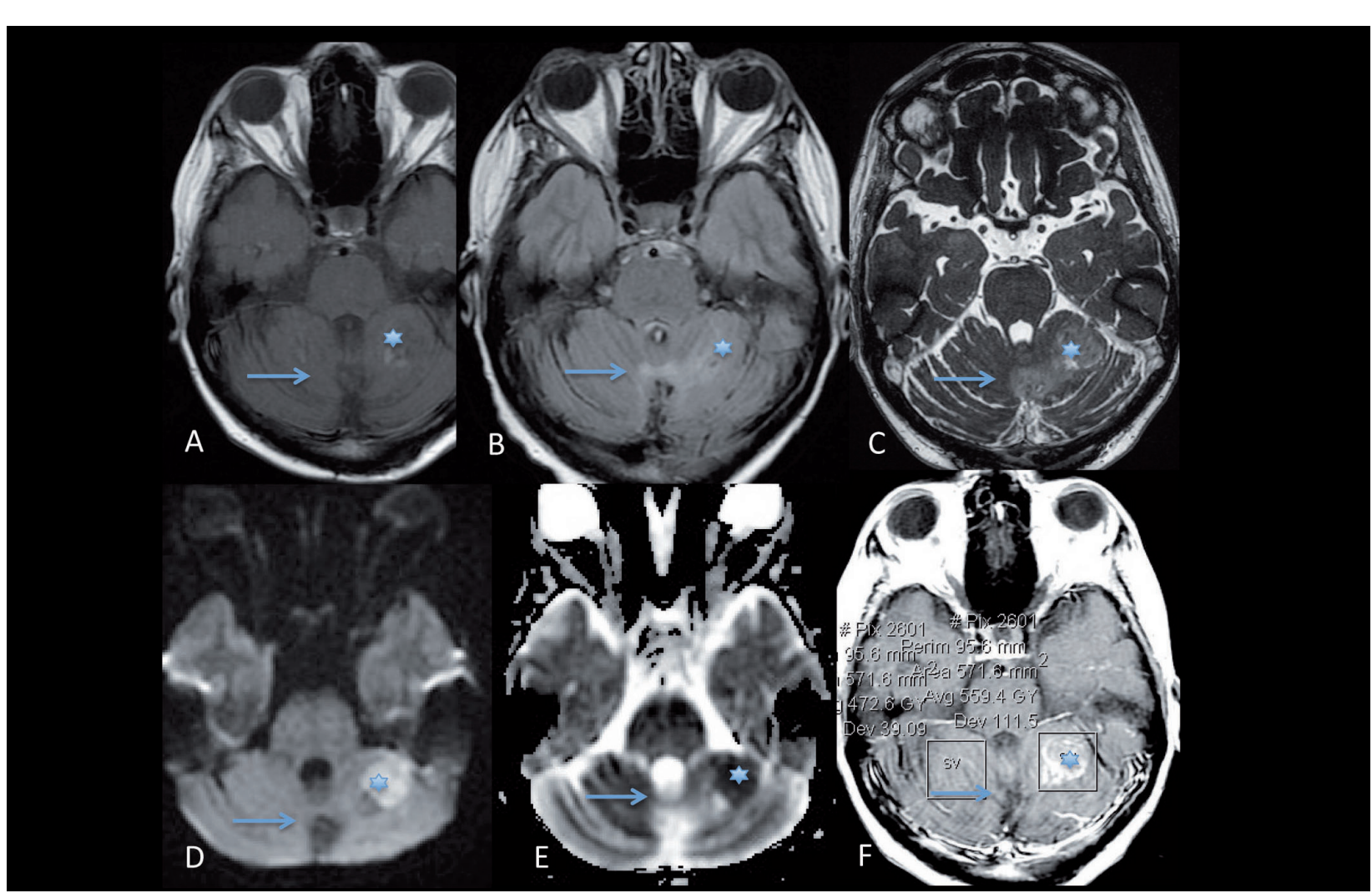

Figure 3. Simple and contrast magnetic resonance of the brain following stem cell transplantation showing focal relapse (treated with radiosurgery). A.TIW B.FLAIR C.T2W D.DWI E.ADC F.TIW contrast 


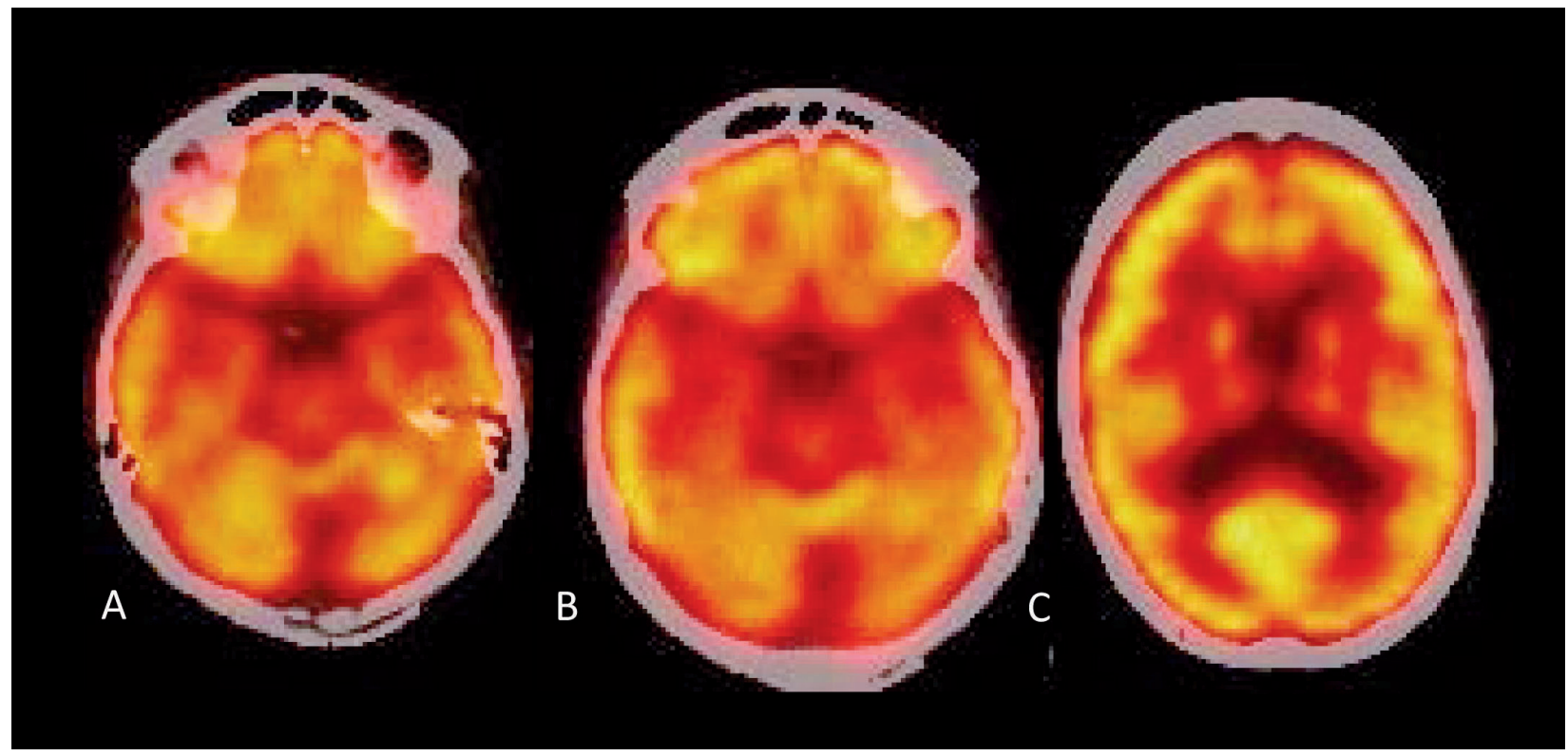

Figure 4. FDG/PET 9 months after stem cell transplantation showing a focal tumour relapse (treated with radiosurgery). $A, B, C$. PET post-transplant with signs of hyper-metabolic lesion corresponding to tumour relapse.

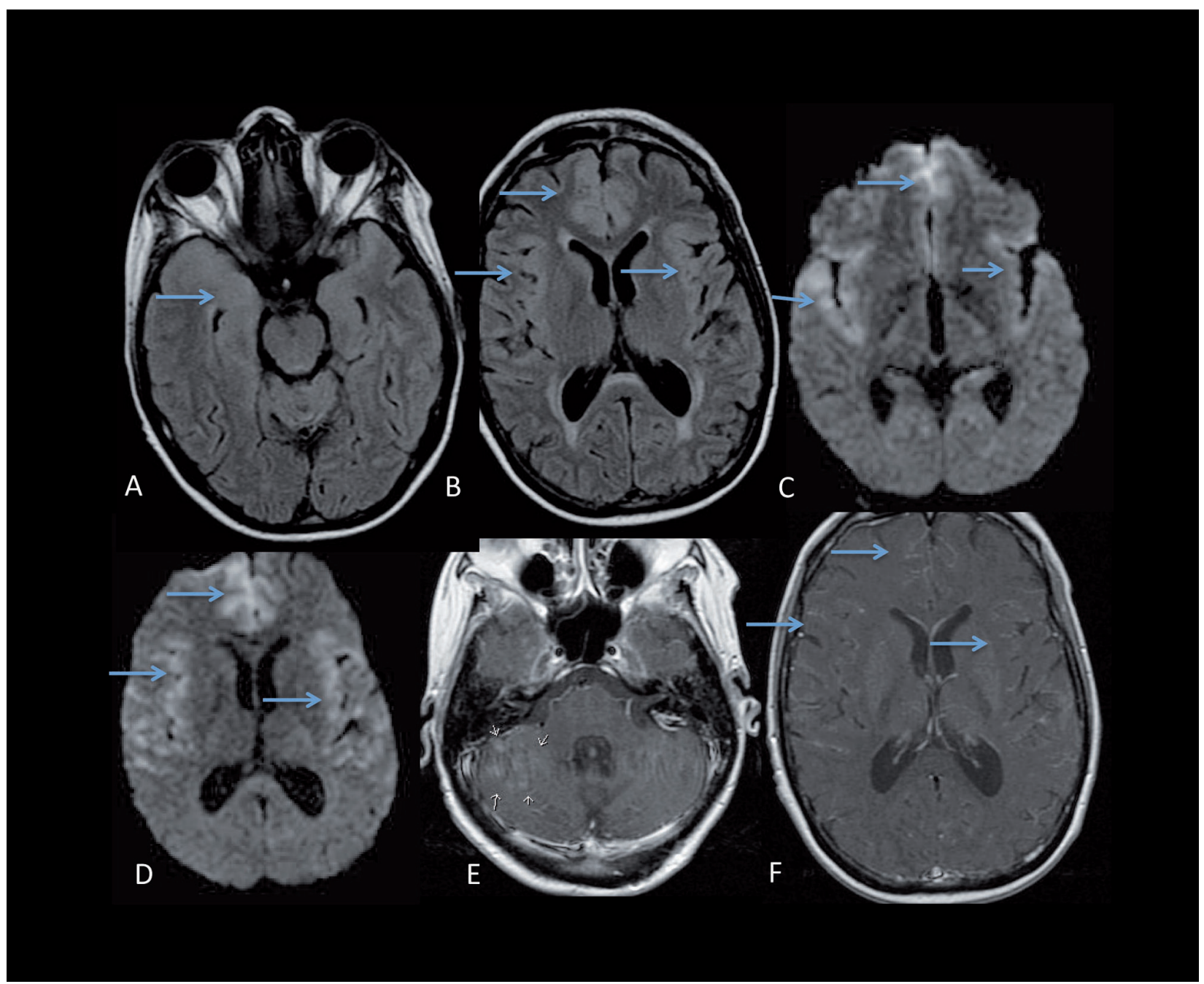

Figure 5. Simple and contrast magnetic resonance of the brain 4 months after radiosurgery, showing leptomeningeal metastatic lesions. A. FLAIR B. FLAIR C. DWI D.DWI E.TIW contrast F. TIW contrast

Axial cross-sections showing areas of signal alteration, with áreas of altered difusión similar to that observad in the initial relapse done with contrast (arrow). 


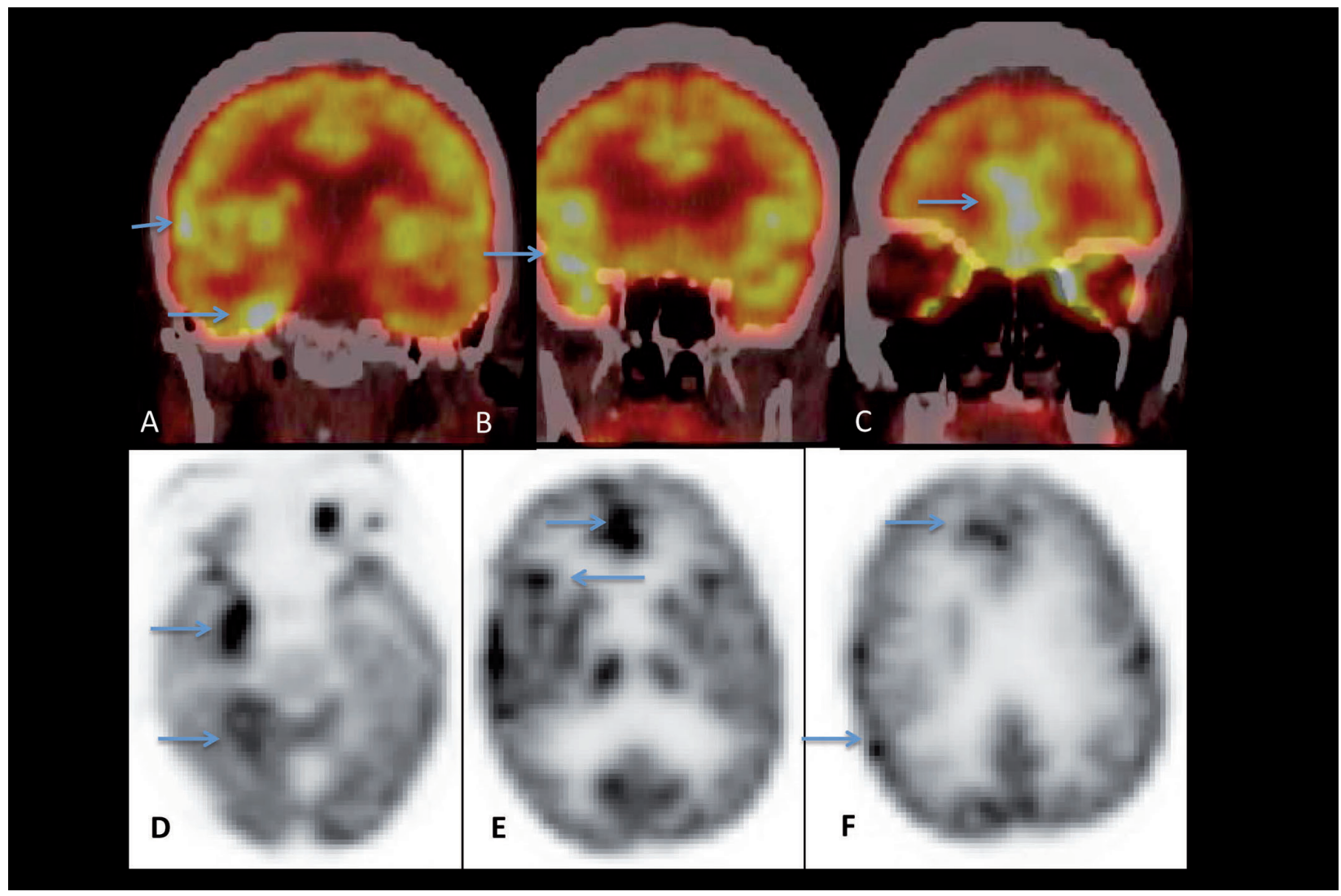

Figure 6. PET-FDG post-radiosurgery with metastatic lesions.

Coronal and axial cross-sections showing areas of increased metabolism (arrows) consistent with leptomeningeal dissemination

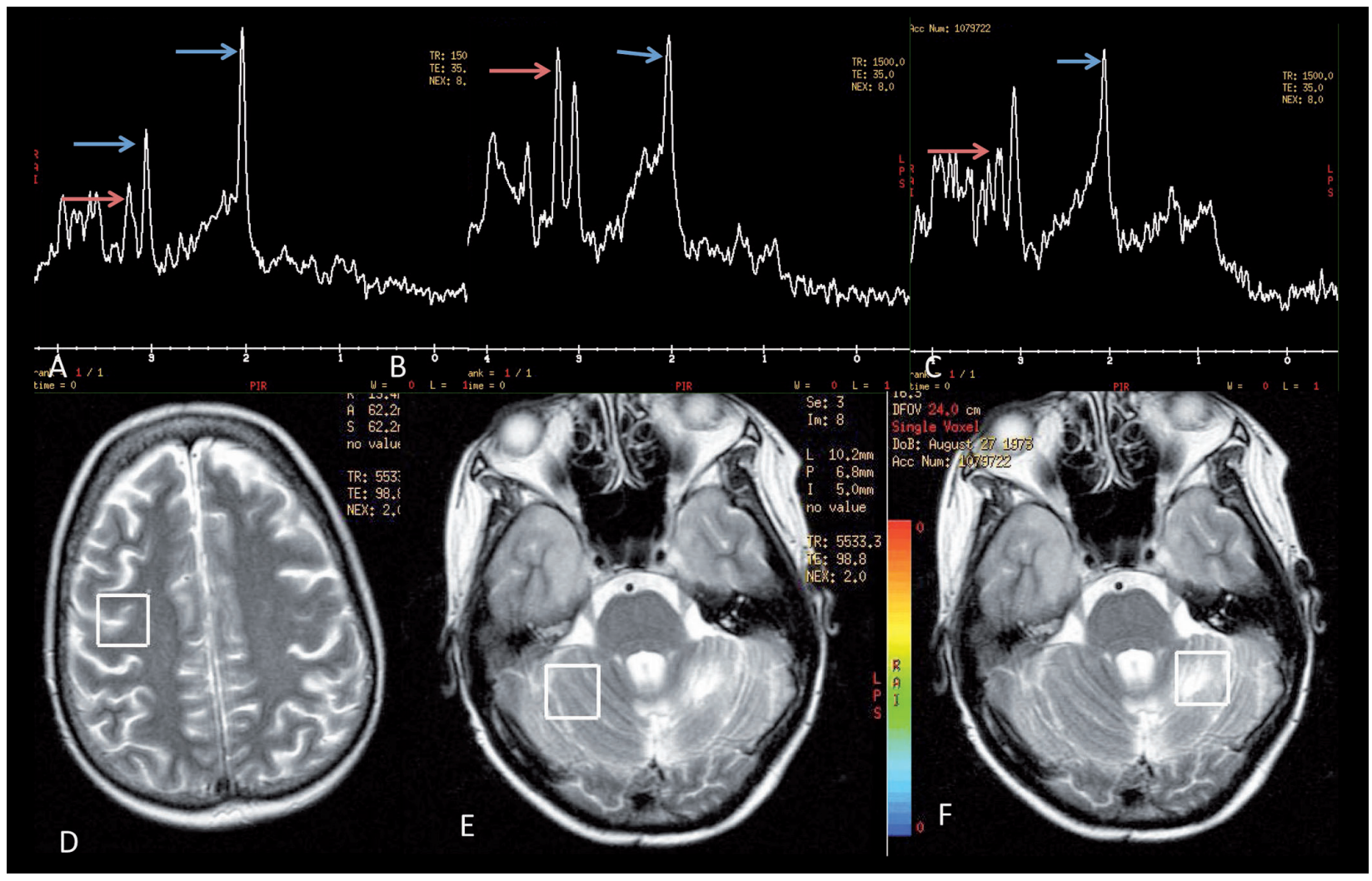

Figure 7. MR spectroscopy showing the respective choline (Cho), creatine ( $C r$ ) and $N$ acetyl aspartate (NAA) peaks in D, E and F (where voxels were taken) and in A, B and C. $A$ and $D$ show normal tissue with high NAA peak (blue arrow) and the expe
and $F$ show a drop in both peaks, probably due to post-treatment changes 


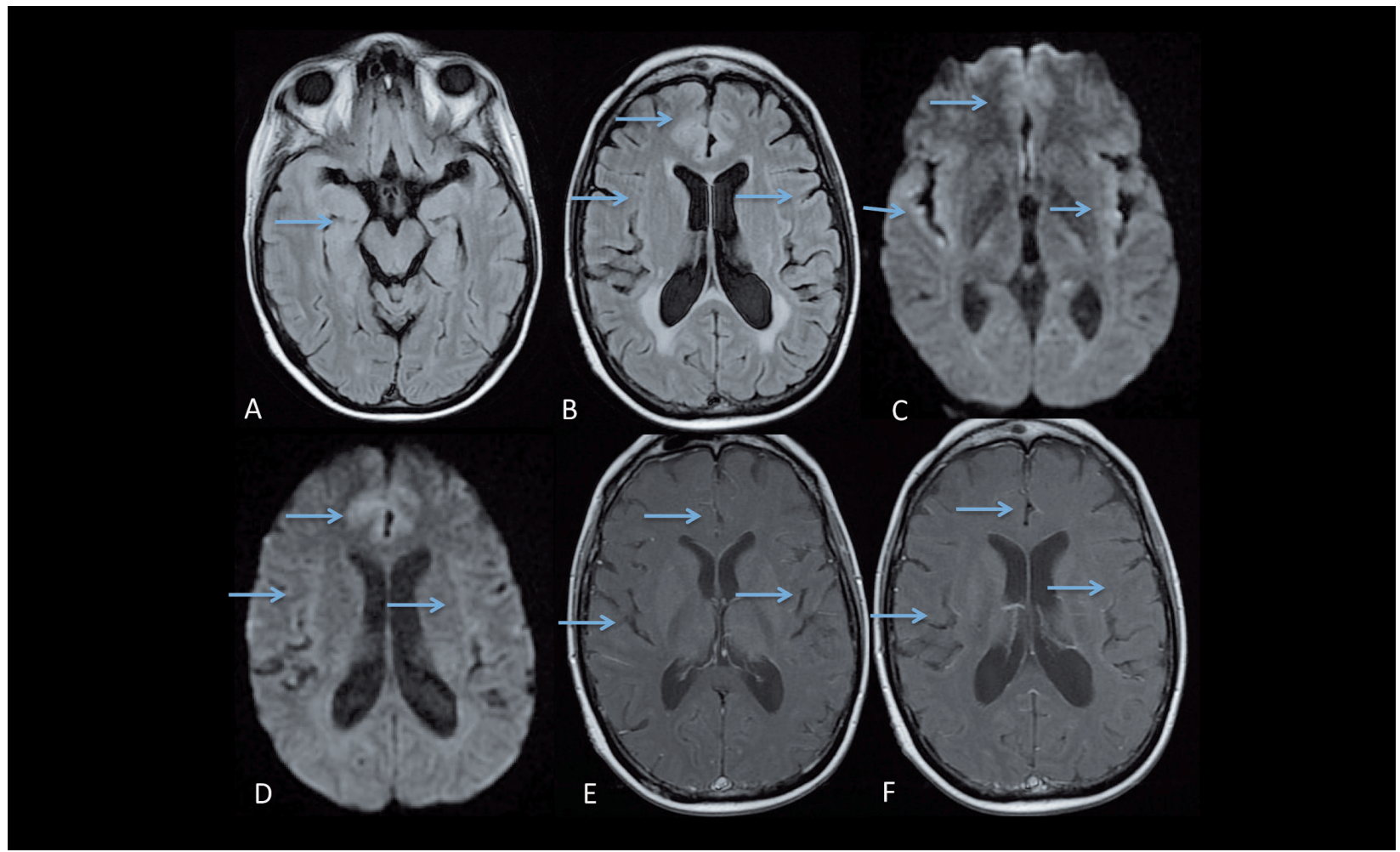

Figure 8. Simple and contrast MR of the brain 4 weeks after starting vismodegib.

Axial cross-sections showing reduced size of areas of signal alteration, with reduced alteration regarding (almost complete) disappearance of diffusion done with contrast (arrows).

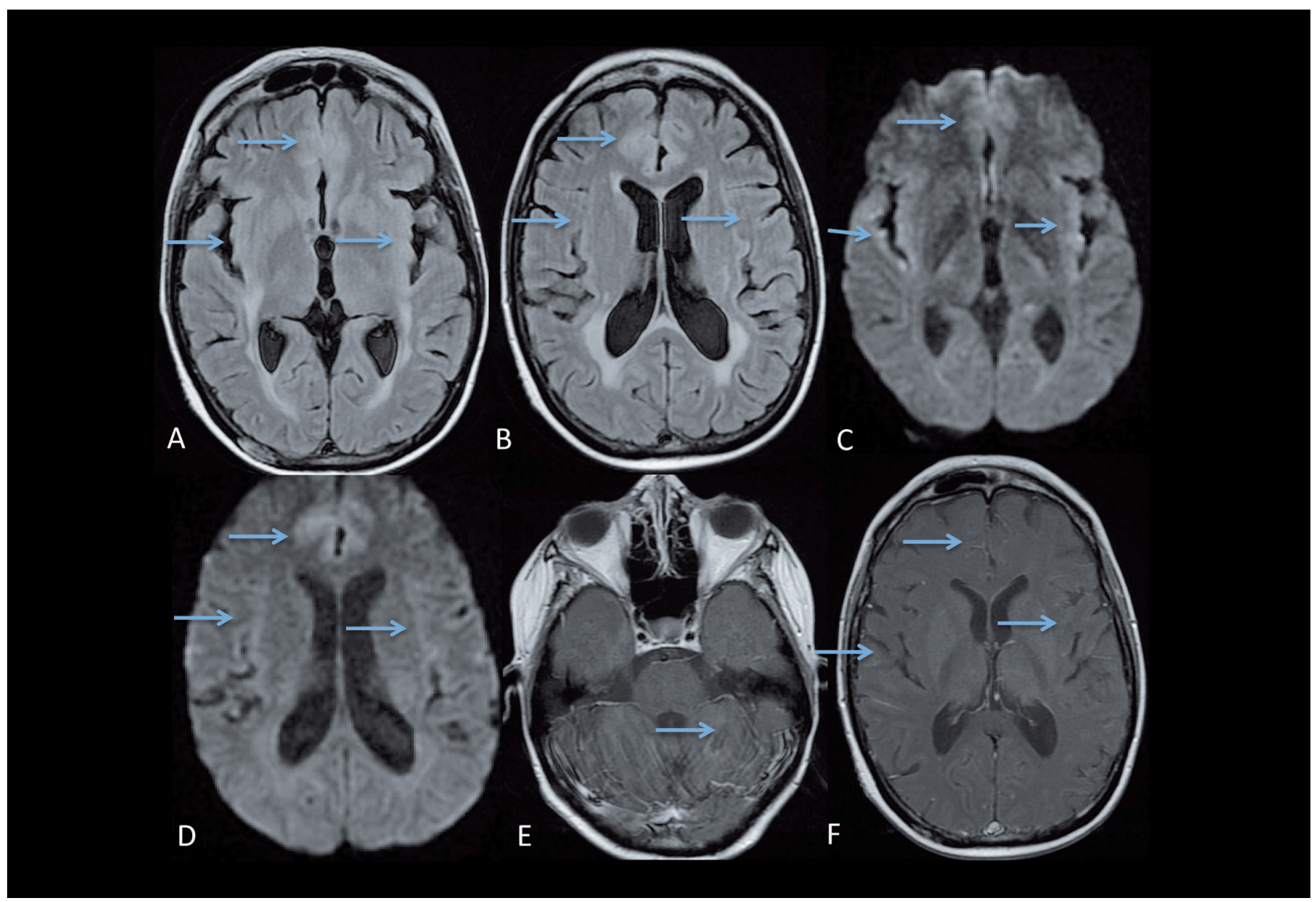

Figure 9. Simple and contrast MR of the brain 10 weeks after starting vismodegib.

A.FLAIR B.FLAIR C. DWI D.DWI E.TIW contrast F.TIW contrast

lesions having a metastatic appearance (arrows). 


\section{References}

1. Dolecek TA, Propp JM, Stroup NE, Kruchko C. CBTRUS statistical report: primary brain and central nervous system tumors diagnosed in the United States in 2005-2009. Neuro Oncol. 2012;14 Suppl 5:v1-49.

2. Rudin $\mathrm{CM}$, Hann $\mathrm{CL}$, Laterra J, Yauch RL, Callahan CA, Fu L, et al. Treatment of medulloblastoma with hedgehog pathway inhibitor GDC-0449. N Engl J Med. 2009;361(12):1173-8.

3. Shonka N, Brandes A, De Groot JF. Adult medulloblastoma, from spongioblastoma cerebelli to the present day: a review of treatment and the integration of molecular markers. Oncology (Williston Park). 2012;26(11):1083-91.

4. Atwood SX, Chang AL, Oro AE. Hedgehog pathway inhibition and the race against tumor evolution. J Cell Biol. 2012;199(2):193-7.

5. Watson S, Serrate C, Vignot S. Voie de signalisation Sonic Hedgehog : du développement embryonnaire aux thérapies moléculaires ciblées. Bulletin du Cancer. 2010;97(12):1477-83.
6. Ruch JM, Kim EJ. Hedgehog signaling pathway and cancer therapeutics: progress to date. Drugs. 2013;73(7):613-23.

7. Chen JK, Taipale J, Cooper MK, Beachy PA. Inhibition of Hedgehog signaling by direct binding of cyclopamine to Smoothened. Genes Dev. 2002;16(21):2743-8.

8. LoRusso PM, Rudin CM, Reddy JC, Tibes R, Weiss GJ, Borad MJ, et al. Phase I trial of hedgehog pathway inhibitor vismodegib (GDC-0449) in patients with refractory, locally advanced or metastatic solid tumors. Clin Cancer Res. 2011;17(8):2502-11.

9. Gajjar AJ, Amar J. A prospective phase II study to determine the efficacy of GDC 0449 (vismodegib) in adults with recurrent medulloblastoma (MB): A Pediatric Brain Tumor Consortium study (PBTC 25B). J Clin Oncol. 2013; suppl: abstr 2035.

10. Weiss GJ, Korn RL. Metastatic basal cell carcinoma in the era of hedgehog signaling pathway inhibitors. Cancer. 2012:118(21):5310-9. 